\title{
Comprehensive backup protection for electrical networks
}

\author{
V. Silbermann \\ Department Transmission \& Distribution, \\ Fichtner Consulting Company, Germany
}

\begin{abstract}
Today, the reliable tripping of faults in the networks by failure of main protection is normally performed with the help of local backup protections, duplicating the principles of main protection. It provides redundancy only in the equipment and cannot trip complicated faults by the inability of main protection to operate due to principle reasons. The usage relays from different manufacturers for these duplicated protections helps only partially because both protections are by faults in the same conditions and act in accordance with the same principles. The damage of protected equipment in this case can be prevented by using the comprehensive remote backup protection. The most important difference between local and remote backup protections is that the remote backup protection acts by faults in the networks under completely different conditions compared to the main and local backup protections. These substantial differences can be used only by correct selection of principles and settings of remote backup protections. This paper reports the principles of performance of such comprehensive remote and some kinds of local backup protections separately for HV overhead and cable lines as well as the principles of selection of characteristics and calculation of settings for corresponding backup protections.

Keywords: failure or inability of main protection, local and remote backup protections.
\end{abstract}

\section{Introduction}

The main task of modern relay protection systems is the reliable fast tripping of any faults in the networks in order to keep the dynamic stability of modern 
electrical systems. However, there are many causes that can lead to the failure of different protections and these causes shall be considered when designing the reliable protection systems, especially for HV networks and auxiliary cable networks of huge power plants. Today, the installation of backup protections is normally performed to compensate for the possible failures of main protection. These backup protections have two incompatible tasks: to guarantee the fast tripping of damaged protected object by failure of main protection and to keep the full selectivity to non-damaged objects in the observed network. For the above reasons, the elaboration of such comprehensive backup protections is a relevant objective when designing protection systems.

\section{Necessary definitions}

The following paragraphs of IEC Standard 60050 (448) [1] describing the functions of the main protection and two kinds of backup protection mentioned above as well as the requirements for these protections shall be considered when designing the reliable protection systems. The $\S 448-11-13$ describes the main protection as "protection expected to have priority in initiating fault clearance or an action to terminate an abnormal condition in a power system" with the note, that "for a given item of plant, two or more main protections may be provided." $\S 448-11-14$ describes the backup protection as protection "which is intended to operate when a system fault is not cleared, or abnormal condition not detected, in required time because of failure or inability of other protection (all italics in the text are of author) to operate or failure of the appropriate circuit-breaker(s) to trip" with the note that "the backup protection may duplicate the main protection or may be intended to operate only if the main protection system fails or is temporarily out of service." The $\S 448-11-16$ of standard [1] describes the so called "substation local backup protection" as protection "which is energized from instrument transformers located within the same substation as the corresponding main protection and not associated with the same primary circuit". In other words this definition describes the protection which is installed by income circuit-breakers or coupler (section) circuit-breaker of the same substation (the first sub-system of backup protections). And the $\S 448-11-17$ of standard [1] gives the definition of the second sub-system of backup protections - the so called "remote backup protection", i.e. protection "located in a substation remote from that substation in which the corresponding main protection is located". Additionally $\S 448-11-9$ of standard [1] defines the so called "unit protection" as "a protection whose operation and section selectivity are dependent on the comparison of electrical quantities at each end of the protected section" and $\S 448-11-10$ of standard [1] defines so called "non-unit protection" as "a protection whose operation and section selectivity are dependent on the measurement of electrical quantities at one end of the protected section by the measuring relays and, in some cases, on the exchange of logic signals between the ends" with the note that "the section selectivity of non-unit protection may depend upon its setting, particularly with regard to time." 


\section{Formulation of a problem}

Consequently, most of the time the possible failures of the main protection are compensating by the installation of second independent main protections (usually additional unit protection for each end of protection section). The independence of these duplicated protections, usually called Main1 and Main2 protections, is ensured by the requirement that they are to be produced by different manufactures which use different apparatus principles of their performance. Additionally, these protections are designed such that they have two independent chains of their performance - from instrument transformers to tripping circuits. They are supplied from different DC sources, connected to different cores of current transformers, to act on different coils of circuit breaker etc. This way it seems that it is possible to carry out the perfect system of relay protection, which functions reliably by the requirement to operate and the existence of one undetected failure in any element in the corresponding protection chain.

The trouble spot in this technical solution is not only the fact that both duplicated protections are due to the faults in the protected object in the same conditions, e.g. they can both fail by damage of accumulator battery at the substation of their installation, but also the fact that they typically use the same protection philosophy, e.g. they both are line differential protections. This same philosophy can lead to the inability of both protections to operate by complicated faults mentioned in the standard [1]. Apart from that, it is often the case that by erection of new and re-construction of existing objects it is impossible to install the second main protection on the object, which belongs to another possessor. And in the low and middle voltage networks as well as in the substations connected to OHL via branch lines it is very expensive and often even impossible to install two independent protections for each object. In this case, the damage of the protected equipment can be prevented only by using the comprehensive remote backup protection. The most important difference between the local and the remote backup protection is that the remote backup protection operates under completely different conditions than main and local backup protections, when the fault occurs, and the remote backup protection is based on a fundamentally different protection philosophy. But these substantial differences can be used only by the correct selection of principles and settings of remote backup protections.

The purpose of this paper is to discuss the specialties of performance of such backup protections separately for HV overhead and cable lines as well as the principles of selection of characteristics and calculation of settings for corresponding backup protections. Very interesting considerations about the topicality and the possible performance of different backup protections were given by Petrov [2] and this paper will also polemize with it in part. All further explanations will be written in designations of standard [1].

One additional common remark is that, the so called (n-1) principle or principle of "one undetected failure" given e.g. by Lothar [3] has to be extended to the performance of the local as well as of the remote backup protection. It 
means that the whole relay protection system shall ensure the reliable tripping of any fault in the protective grid when this fault occurs and one undetected failure of any protection (any circuit breaker) in this system. This principle ensures the reliability of functioning of the equipment (in our case of the relay protection) on the one hand and minimizes the costs on the other hand: multiple reservations of relay protection functions lead to the unnecessary cost improvement as well as to difficulties in understanding of the principles of performance of backup protections. The latter leads to essential difficulties when it comes to the calculation of selective relay protection settings.

\section{The ways to performance of comprehensive backup protections: discussion}

From standard [1] follows that the reliable function of both local and remote backup protection can be guaranteed if these protections will react merely to the parameters (current, voltage, impedance etc.), measured or calculated on the site where this protection is installed. Only such measuring (the so called backup protection autonomy) can guarantee the operational freedom of the backup protection e.g. it excludes the possibility of the failure of the backup protection by full disappearance of direct current on the reserved protection. It means also that backup protections may not use any signal from remote objects, i.e. any backup protection has to be carried out as time-delay protection only. These time delays can depend on the parameters, which backup protection measures, or they can be also carried out with definite time delay for all fault currents exceeding the protection current setting.

It is also relevant to note that despite the necessity of performance of comprehensive backup protections, which was argued in standard [1] as well as by Petrov [2] and by Fabricant [4], every action of both local and remote backup protections means the tripping of additional objects (consumers). Because of that, the probability of any unwanted operation of the backup protection, i.e. an operation which is formulated in standard [1] as "the operation of a protection either without any power system fault or other power system abnormality, or for a system fault or other power system abnormality for which that protection should not have operated", shall be excluded absolutely. It means that the performance of the backup protection with partial selectivity, as it was proposed by Petrov [2], is inadmissible. These protections shall be coordinated both with parameter settings (current, voltage, impedance etc.) and with time settings of main protections which are backed.

It is also necessary to discuss the Petrov's statement [2], that the full selectivity of two current protections with time-current characteristics, e.g. ABB logarithmic characteristic $\mathrm{t}=5.8-1.35 * \ln \left(\mathrm{I}_{0} / \mathrm{I}_{\mathrm{N}}\right)$ is possible even for the same settings of these protections. The Petrov's calculation [2] have shown that by the conditions above the time delay between these protections equals $\Delta t \approx 0.4 \mathrm{sec}$, i.e. these protections have full selectivity for an acceptable tripping time. But this calculation did not regard the existing setting errors $\pm(2-5) \%$. It means that the reliability factor $K_{r}$, which increases the value $\Delta t$, shall be inserted into the 
calculation. i.e. in the equation showing the coordination of the backup protection " $m$ " with failed protection " $n$ ":

$$
\Delta t=1.35 * \ln \left(\frac{I_{0 n}}{I_{0 m}} * \frac{I_{N m}}{I_{N n}}\right)
$$

given by Petrov [2], ( $I_{0}-$ the fault currents, flowing via corresponding protections, $I_{N}$ - settings of these protections, $I_{N m}=I_{N n}$ ). As a result, eqn (1) can now be written as follows:

$$
\Delta t=1.35 * \ln \left(K_{r} * \frac{I_{0 n}}{I_{0 m}} * \frac{I_{N m}}{I_{N n}}\right)
$$

Even by minimal reliability factor $K_{r . \min }=1.05$ i.e. by setting the value of error of modern protections $\pm 2 \%$, the distribution factor (relation of currents in the damaged and non-damaged lines) shall be $I_{0 n} / I_{0 m} \geq 1.28$ instead of the usual distribution factor $I_{0 n} / I_{0 m} \geq 1.35$, given in [2] in order to receive $\Delta t=0.4 \mathrm{sec}$ acceptable for the tripping time of the backup protection when faults are close to the place of installation of this protection. $B y K_{r}=1.11$ (for usual set-tings error $\pm 5 \%$ ) the distribution factor shall be $I_{0 n} / I_{0 m} \geq 1.21$. Such conditions do not exist in every network. Because of that, the usage of current backup protections with dependent (logarithmic) time-current characteristics is restricted and can be recommended only for local backup protections in simple networks with motors (first of all in auxiliaries of power plants) where the distribution factors always exceed 1.2. The usage of distance protections can be recommended for remote backup protections in the HV networks and double-fed MV networks.

\subsection{Performance of comprehensive local backup protection}

The performance of the comprehensive local backup protections in the cable auxiliary networks described above is absolutely necessary because the failure of any feeder protection when the local backup protection is absent or has insufficient sensibility to the remote short circuits, leads to an ignition of a damaged cable and following burning-out of the whole cable network. Such a backup protection shall be installed at the network income and meet two controversial conditions: it shall have the necessary sensitivity to remote faults in the cable network and at the same time it shall be offset from the start current of any motor as well as from the current flowing via income by self-start of the motor group supplied from this income. However, the problem arises that the magnitude of the start current and especially self-start current can be significantly larger that the magnitude of the remote fault current.

The simplest method to differentiate between the start of the motor or the self-start of the group of motors and remote 3-phase fault in the cable is to measure or to calculate the angle between the current which flows via the local 
backup protection (income circuit breaker) and the voltage on the bus which supplies this cable network. This method is described in details in German patent [5] and it can be demonstrated on the example of a $6 \mathrm{kV}$ cable network with motors. It is well known that the angle between the start current of any $6 \mathrm{kV}$ motor and the bus voltage normally exceeds 75 degrees. Let us assume that current flows via income equal $1.2 * I_{R}\left(I_{R}\right.$ is the rated current of income supplying the protected cable network) and the rest of load supplied from this 6 $\mathrm{kV}$ bus is active. Then in this worst case the angle between the income current and the bus voltage at the start of this alone motor always exceeds the value of 50 degrees. Given the remote 3-phase fault in the cable, for the same value of the income current even for $6 \mathrm{kV}$ copper cables with cross-section area of $185 \mathrm{~mm}^{2}$ this angle is less than 40 degrees. Although the values of the motor start angle and cable cross-section area in $0.4 \mathrm{kV}$ networks differ from values in $6 \mathrm{kV}$ networks, the same difference between current angles can also be demonstrated for $0.4 \mathrm{kV}$ cable networks with motors. This phenomenon allows to differentiate between these two operation conditions and to trip income circuit breaker only when faults in cables occur, and to block such tripping at the start of any motor or at the self-start of motor groups (e.g. by voltage drop as a result of short circuits in the supplied network). This means that the comprehensive local backup protection whose sensitivity is restricted only by the short circuit current value $I_{S . C .}=1.2 * I_{R}$ can be installed in these cable networks. Such a condition is practically observed in all cable networks with motors.

When designing the comprehensive local backup protection it shall be also considered that cable feeders are normally protected with relays or fuses with IDMT characteristics to guarantee the reliable start of protected motors. It means that the backup protection shall also have the IDMT characteristic, but the problem is that this characteristic shall be coordinated not only with IDMT characteristics of outgoing feeder with the maximally rated current but also with thermal characteristics of the damaged cable with the smallest cross-section area. Only such a coordination can prevent the fire in the cable network when fault in this cable occurs and its protection fails. To avoid the possible misunderstanding it has to be clarified that the fuse failure should be read as the wrong installation of fuses with enhanced rated currents instead fuses with rated currents corresponding to ratings of the protective cable and motor. The melting time of such a fuse, when short circuit occurs, exceeds the previously estimated time and this damaged cable will ignite before the fuse melts. Such errors of the operating staff were already recorded in some countries.

The most significant problem, when such reasonably large operative time delays of the local backup protection occur, is however, that it is necessary to take into account the phenomenon of the thermal reduction of the short circuit current in the damaged cable [5]. This phenomenon occurs due to the increase of resistance of the cable core as a result of its heating by the short circuit current. It will lead to the inability to trip this fault by protection with the usual IDMT characteristic, because the current value measured by this protection reduces faster than the elapsing time, which corresponds to the initially measured current. Such a reduction can be described with equation given by Oulianov [6]. This 
equation connects the values of the current temperature of the cable core $v$ in Kelvin degrees $\left({ }^{\circ} \mathrm{K}\right)$, the running time of fault existence $t$ in seconds and the cross-section area of damaged cable $S$ in $\mathrm{mm}^{2}$ :

$$
\begin{gathered}
I_{i}^{2} * t= \\
=S * \frac{c^{*} \gamma}{\rho} *\left\{\frac{R_{K}^{2}}{Z_{k}^{2} *\left(1+\alpha * v_{i}\right)} *\left[\left(v-v_{i}\right)+\frac{\alpha}{2} *\left(v^{2}-v_{i}^{2}\right)\right]+\right. \\
\left.+\frac{\left(1-\frac{R_{K}^{2}}{Z_{K}^{2}}\right) *\left(1+\alpha^{*} v_{i}\right)}{\alpha} \ln \frac{1+\alpha^{*} v}{1+\alpha^{*} v_{i}}\right\}
\end{gathered}
$$

Here:

$I_{i}$ - initial value of effective short circuit current in Amp,

$v_{i}$ - initial temperature of cable core in ${ }^{\circ} \mathrm{K}$,

$R_{K}$ - resistance of $1 \mathrm{~m}$ of damaged cable by initial temperature in $\Omega / \mathrm{m}$,

$Z_{K}$ - impedance of $1 \mathrm{~m}$ of damaged cable in $\Omega / \mathrm{m}$,

$\rho$ - specific electrical resistance of damaged cable in $\mathrm{mm}^{2} * \Omega / \mathrm{m}$,

$c$ and $\gamma-$ specific heat capacity (in $\mathrm{W} / \mathrm{g}^{*}{ }^{\circ} \mathrm{K}$ ) and density (in $\mathrm{g} / \mathrm{cm}^{3}$ ) of core material of damaged cable,

$\alpha$ - temperature coefficient of cable core materials (in $1 /{ }^{\circ} \mathrm{K}$ ).

Assuming that the temperature of insulation ignition is a constant value for a given cable and inserting into eqn (3) this value $v_{\text {core }}=v_{\text {insulation ignition instead of }}$ the current value $v$, eqn (3) can now be written for each cable as

$$
I_{i}^{2} * t_{\max }=C=\text { const }
$$

i.e. regardless of the complicated change of the cable core temperature when a short circuit occurs, the period $t_{\max }$ where temperature of cable core (and cable insulation) rises up to the ignition temperature of the cable insulation is also a constant value for each cable for a given value of the initial short circuit current in cable $I_{i}$.

Consequently, in order to exclude the influence of thermal reduction of short circuit current in the damaged cable on the operation of the local backup protection it was proposed in patent [5] to calculate the value $C$ for the cable with the minimal cross-section area and to put this value as the setting into local backup protection. Whenever the fault in the cable network occurs, i.e. when the angle between the income current and bus voltage is less than 40 degrees, the local backup protection measures the value $I_{i}$, flowing through income circuit breaker, calculates from values $I_{i}$ and $C$ the maximal possible value of tripping time to prevent the cable ignition $t_{\max }$ and saves it in the protection. After elapsing of this time the protection measures the flowing current once more and 
if the current value exceeds $1.2 * I_{\text {rated }}$, (it estimates as the failure of the cable protection) the local backup protection trips the income circuit breaker. It was shown by the author [7] that the time $t_{\max }$ calculated for the cable with minimal cross-section area will be also selective to IDMT characteristic of protection of the cable with the maximal cross-section area if the rated current of motor fed via this feeder is less than $1 / 4$ of the rated current of the income circuit breaker. This relation is always present in the networks with motors to assure a reliable start of the largest motor, i.e. the principles above allow to perform the reliable and selective local backup protection for the simple networks.

\subsection{Performance of comprehensive remote backup protection}

The usage of the distance protections with polygonal characteristics as the backup protection when both protection against multiphase faults and protection against single-pole faults fails, is the most reasonable way for the performance of remote backup protections. It is only necessary that characteristics of zones used for the backup functions could be displaced into the $1^{\text {st }}$ quadrant of the complex plane without restrictions.

Let us exemplify it as follows. Envisage the fault occurring at the end of line L2 of the network consisting of two lines which feed the load of substation " $n$ ", fig. 1. When of the protection at the substation " $n$ " fails, this fault shall be eliminated by the operation of distance protection of line L1 at substation " $m$ ", i.e. the phasor of fault impedance measured at the substation " $m$ " shall be found within the characteristic of the backup zone in the complex plane. But when the load phasor $Z_{\text {Load }}$ is offset, the typical characteristic passed through the origin of complex plane (broken line in fig. 1) does not allow to assure the necessary sensitivity to arcing short circuit currents which can be replaced in the $4^{\text {th }}$ quadrant of the complex plane as it was shown by Fabricant [4]. It is also very difficult to coordinate the characteristic shown by the broken line in fig. 1 with characteristics of the distance protections lines adjacent to the substation " $p$ " etc.

All these difficulties can be easily overcome if the characteristic of backup zone is displaced into the $1^{\text {st }}$ quadrant of the complex plane so as to offset the impedance of the line " $m-n$ " $Z_{L 1}$ as it is highlighted by the red full line in fig. 1 .

The parameters of such a displaced characteristic shall be calculated analogously to the parameter of the distance zone at substation " $n$ " which shall be backed, considering of course the necessary factors of reliability $K_{r}=1.15$. This factor considers the maximal possible setting error and avoids to make the tripping area of backup distance protection larger than tripping area of backed distance protection. Apart from that, this characteristic can be an out-of-position horizontal axis $R_{n}$ in the complex plane, i.e. this characteristic will respond to the complex phasor of arcing fault at line L2 as well as exclude the "hampering effect of connected feeders" described by Petrov [2]. In other words this way allows the remote backup protection to perform the function of the local backup protection "carried" from substation " $n$ " (axes $R_{n}$ and $X_{n}$ in fig. 1) to the remote substation " $m$ " (axes $R_{m}$ and $X_{m}$ in fig. 1).

The displacement of the distance characteristic can also be used to back up the failure of protections installed at the transformer connected to $\mathrm{OHL}$ via 

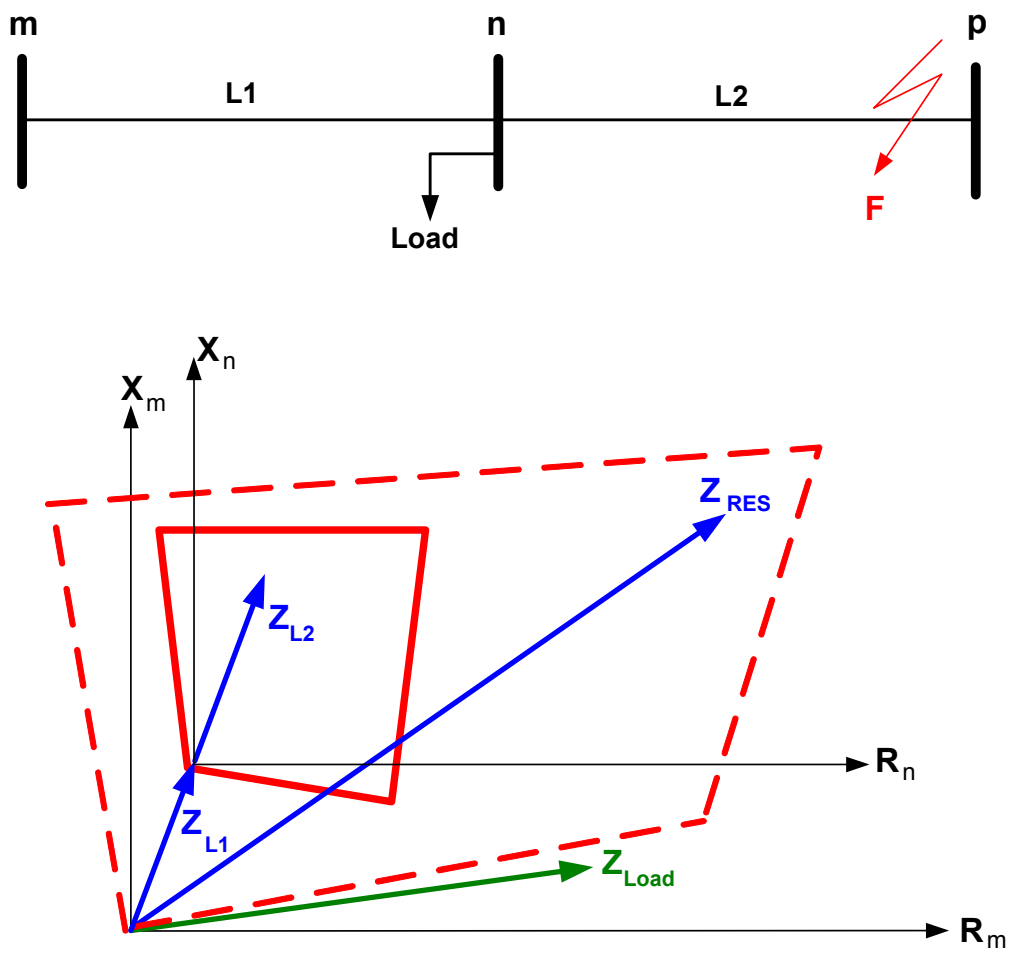

Figure 1: $\quad$ Example of typical distance characteristic (with a broken line) and proposed distance characteristic for backup protection of line n-p.

branch line. The impedance of transformers with $\mathrm{HV} \geq 110 \mathrm{kV}$ can be practically observed as inductive, i.e. this phasor can be calculated with an angle about $\varphi_{T r}=90^{\circ}$. It means that the characteristic of the backup zone of protection installed at substation " $m$ " (fig. 2), can easily offset both the phasor of impedance of line L2 with angle $\varphi_{L}=(65-75)^{\circ}$ and the phasor of load impedance $Z_{\text {Load }}$ with angle less than $30^{\circ}$. The modern distance protections have an angle setting error which does not exceed $\Delta \varphi= \pm 2^{\circ}$. Hence such an offset can be carried out without any difficulties. The same criteria are also valid for the selection of backup characteristics of the distance protection installed at the substation " $n$ ".

Hence it can be recommended the usage of conventional 5-zone distance protection for the performance of the comprehensive remote backup protection at the OHLs $110-750 \mathrm{kV}$. Along with it, the first three zones shall be used for the protection of "own" line with the usual principles of selection of their settings and characteristics. The remaining two zones with polygonal characteristics displaced into the $1^{\text {st }}$ quadrant of the complex plane shall be used as the remote backup protection for faults at adjacent lines and failures of corresponding protections. One of these zones shall be used for the performance of the backup 


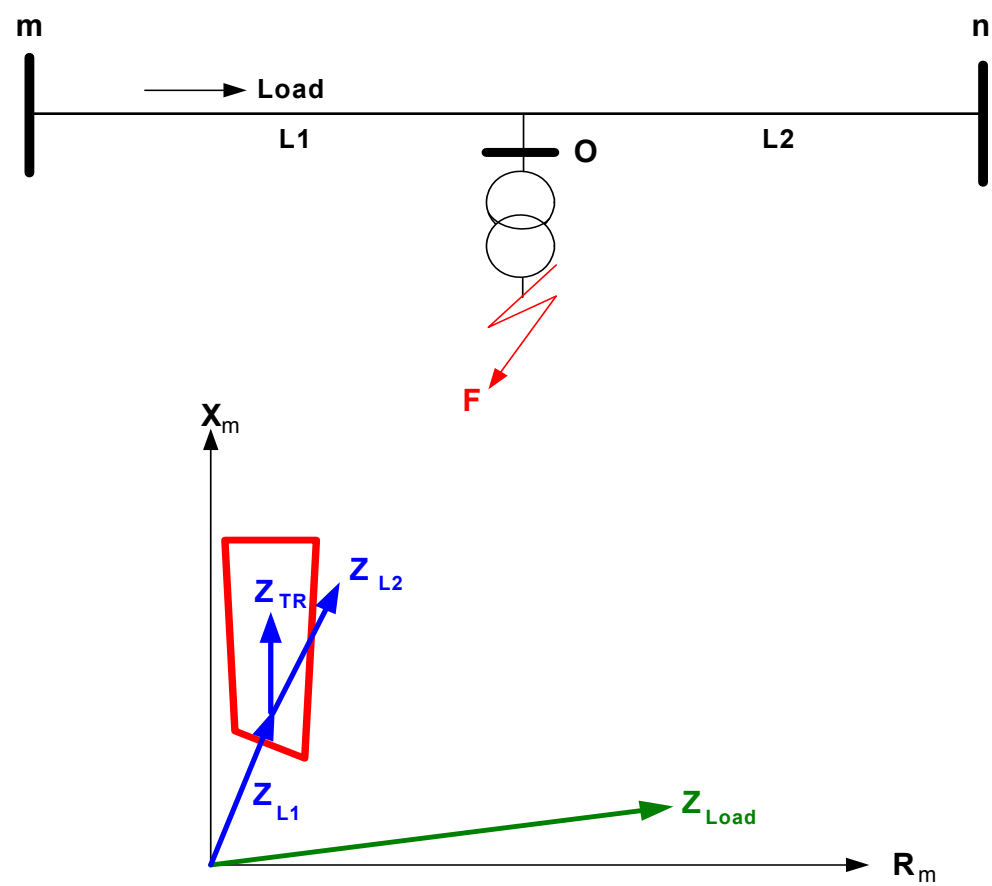

Figure 2: Example of distance characteristic for backup protection of transformer connected to OHL via branch line.

protection by multiphase faults and the remained zone shall be used for backup protection by single-pole faults. Both these zones foreseen for the remote backup protection shall be fully coordinated with the distance zones of adjacent lines which protect the whole adjacent line to keep the (n-1) principle.

Only 2 zones shall be used for line protection if one transformer is connected to this line via the branch line, next 2 zones shall be used for the remote backup function above and the last zone with the characteristic performed in accordance with fig. 2 shall be used to back the failure of transformer protections. The functions of the remote backup protections for parts L1 and L2 of this line will be performed by distance protections of lines adjacent to substations " $m$ " and " $n$ " with characteristics shown in fig. 1.

Although the distance protections produced today do not foresee the displacement of their characteristics into the $1^{\text {st }}$ quadrant of the complex plane, it is not hard to perform such characteristics if such order is available.

\section{Conclusions}

1. It is necessary to differentiate the tasks of the local and the remote backup protections by performance of the backup protection functions. 
2. An appeal to (n-1) principle and to principle of distinguishing of fault currents and currents by motor start due the definition of angle between income current and bus voltage as well as to principle of overcoming of thermal reduction of short circuit current in the damaged cable makes possible to perform the comprehensive local backup protection for cable networks with motors.

3. An appeal to (n-1) principle and to displacement of distance characteristics into the $1^{\text {st }}$ quadrant of the complex plane makes possible to perform the comprehensive remote backup protection for all kinds of faults at OHLs 110 $750 \mathrm{kV}$ inclusive OHLs with branch lines.

4. By order of the distance protections it is necessary to call upon the manufacturer to guarantee the possibility to displace the distance characteristics into the $1^{\text {st }}$ quadrant of the complex plane at least for three last zones.

\section{References}

[1] IEC Standard 60050 (448), 1995

[2] S.Y. Petrov. The remote backup protection in protection system. Nasha Energetika, (1), pp. 19-21, 2008 (in Russian).

[3] F. Lothar. Zollenkopf vs. (n-1) - Prinzip vs. Kosten - ein Lösungsvorschlag für die optimierte Gestaltung von Netzen, E \& I - Elektrotechnik und Informationstechnik, Wien, (10), pp. 377-379, 2004 (in German).

[4] V.L. Fabricant. Distance Protections, Vys'shaya Shcola, pp. 21 and 44, 1978 (in Russian).

[5] V. Silbermann. Verfahren zum vollselektiven Reserveschutz in Kabelnetzen. German Patent Nr. DE 19741662 A1, 1998 (in German).

[6] S.A. Oulianov. Electromagnetic Transient Phenomena in Electrical Systems, Energiya, pp. 443-447, 1970 (in Russian).

[7] V.A. Silbermann. Relay Protection of Auxiliary Networks in the Nuclear Power Plants, Energoatomizdat, pp. 74-77, 1992 (in Russian). 